\title{
ПЕРЕНОС ЭЛЕКТРОННОГО ВОЗБУЖДЕНИЯ В ХОДЕ КОЛЕБАТЕЛЬНОЙ РЕЛАКСАЦИИ
}

\section{1. Некогерентная горячая передача}

Цель данного сообщения - рассмотреть общие свойства переноса энергии от донора к акцептору в неравновесном (горячем) колебательном состоянии. Такой перенос энергии в [ $\left.{ }^{1}\right]$ был назван горячей передачей (ГП) электронного возбуждения. Наше рассмотрение основано на теории ГП, развитой в $\left[{ }^{1-3}\right]$. Теория ГП возникла как логическое обобщение теории Ферстера [ $\left.{ }^{4}\right]$. В этой теории вероятность переноса электронного возбуждения в неравновесном колебательном состоянии определяется интегралом перекрывания спектра излучения донора со спектром поглощения акцептора. Характерное время колебательной релаксации $t_{\text {рел }} \sim 10^{-11}-10^{-12} c$ обычно гораздо короче времени жизни возбужденного электронного состояния: Поэтому процесс передачи, рассмотренный Ферстером, является, как правило, основным. Однако в тех случаях, когда взаимодействие донора и акцептора $u$ достаточно сильное $\left(u \sim t_{\text {рел }}{ }^{-1}\right)$, перенос возбуждения может с заметной вероятностью произойти еще до окончания колебательной релаксации $\left[{ }^{2,5}\right]$. Полная вероятность переноса возбуждения $w\left(\omega_{0}\right)$, включая перенос как после релаксаций, так и в ходе ее, определяется интегралом перекрывания всего спектра резонансного вторичного свечения (РВС) (т. е. рассеяния, горячей люминесценции (ГЛ) и обычной люминесценции (ОЛ)) донора со спектром поглощения акцептора $x_{A}\left[{ }^{2}\right]$ :

$$
w\left(\omega_{0}\right)=u^{2} \int d \omega \varkappa_{A}(\omega)\left[I_{\text {pacc }}^{D}\left(\omega_{0}, \omega\right)+I_{\text {ГЛ }}^{D}\left(\omega_{0}, \omega\right)+I_{\text {Ол }}^{D}(\omega)\right]
$$

( $\omega_{0}$ - частота возбуждения). Первые два слагаемых в квадратных скобках учитывают ГП, третий член - ферстеровскую передачу энергии.

Следует отметить, что формула (1), как и соответствующая формула теории Ферстера, описывает лишь некогерентный перенос электронного возбуждения. Этот случай представляет особый интерес, поскольку он соответствует таким взаимодействиям донора и акцептора, при которых их индивидуальные свойства заметно не нарушаются. Напротив, когерентный перенос имеет место тогда, когда взаимодействие донора и акцептора настолько сильное, что рассмотрение их как независимых подсистем невозможно. Количественным критерием существования некогерентной ГП является неравенство

$$
t_{\text {рел }}^{-1} \leqslant u<\sigma_{A},
$$

где $\sigma_{A}-$ характерная ширина спектра поглощения акцептора в резонансной области.

Основные свойства ГП:

1) высокая скорость - характерное время ГП $t_{\text {гп }} \sim 10^{-12}-10^{-13} c$;

2) малая потеря энергии при переносе возбуждения с донора на акцеп- 
тор; это свойство ГП особенно важно, так как благодаря ему ГП можно ожидать и в тех случаях, когда обычная передача невозможна из-за нехватки энергии;

3) сильная зависимость вероятности ГП от частоты возбуждения уже в пределах элементарной электронно-колебательной полосы.

Как известно, спектры РВС донора и спектр поглощения акцептора могут быть довольно сложными. Поэтому в общем случае вычислить $w_{\text {гп }}\left(\omega_{0}\right)$ не представляется возможным (но это можно сделать в каждом конкретном случае). Часто, однако, мы имеем дело с системами, в которых спектр поглощения акцептора характеризуется-большой шириной $\sigma_{A} \gg \bar{\hbar} \bar{\omega}(\bar{\omega}-$ средняя частота колебаний) и отсутствнем колебательной структуры. (Это случай сильного и среднего вибронного взаимодействия в акцепторе.) Такой расчет зависимости $w_{\Gamma п}\left(\omega_{0}\right)$ от частоты возбуждения оказывается возможным. Здесь мы рассмотрим указанную зависимость в двух важных случаях:

1. Медленная колебательная релаксация в доноре. Обычно ей соответствуют структурные квазилинейчатые спектры РВС, в том числе ГЛ. В этом случае вероятность ГП по порядку величины равна

$$
w_{\text {гп }} \sim u^{2} t_{\text {релI }} / \sigma_{A} .
$$

Точная вероятность определяется формулой

$$
w_{\Gamma \Pi}\left(\omega_{0}\right)=u^{2} \int x_{A}(\omega) I_{\Gamma Л}^{D}\left(\omega_{0}, \omega\right) d \omega,
$$

что дает сложную зависимость от частоты возбуждения, похожую на зависимость в спектрах возбуждения ГЛ* (с четкими квазилиниями). Однако если вибронное взаимодействие в акцепторе, как и в доноре, достаточно большое, то полная ширина спектра ГЛ $\Delta_{\Gamma л} \simeq 3,5 S_{D}\left(S_{D}-\right.$ стоксовы потери в доноре) велика не только по сравнению с $\hbar \bar{\omega}$, но и по сравнению с шириной спектра поглощения акцептора $\sigma_{A} \sim\left(S_{A} \hbar \bar{\omega}\right)^{1 / 2}$. В таком случае огибающую спектра ГЛ донора в коротковолновой области можно аппроксимировать как

$$
I_{\Gamma Л}\left(\omega_{0}, \omega\right) \sim\left(\omega_{0}^{\prime}-\omega\right)^{1 / 2},
$$

где $\omega_{0}^{\prime}=\omega_{0}-\delta, \hbar \delta-$ потеря энергии на быстром этапе релаксации (который обычно всегда имеется). Подставляя (3) в (2), получим следующую формулу **

$$
w_{\Gamma \Pi}\left(\omega_{0}\right) \sim u^{2} \mathrm{e}^{-x^{2}} \int_{0}^{\infty} d y \sqrt{y} \mathrm{e}^{2 x y-y^{2}}=
$$

$$
=u^{2} \frac{\sqrt{\pi}}{4 \sqrt{2}} \mathrm{e}^{-x^{2}}\left[\frac{\Gamma(1 / 2)}{\Gamma(5 / 4)} \Phi\left(\frac{3}{4} ; \frac{1}{2} ; x^{2}\right)-x \frac{\Gamma(-1 / 2)}{\Gamma(3 / 4)} \Phi\left(\frac{5}{4} ; \frac{3}{2} ; x^{2}\right)\right]
$$

где $x=\left(\omega_{0}-\omega_{A}\right) / \sigma_{A} \sqrt{2}-$ безразмерная частота возбуждения, отсчитанная от максимума поглощения акцептора $\omega_{A}, \Gamma(z)$ - гамма-функция, $\Phi(\alpha ; \gamma ; z)=1+\frac{\alpha}{\gamma} z+\frac{\alpha(\alpha+1)}{\gamma(\gamma+1)} \cdot \frac{z^{2}}{2 !}+\ldots-$ вырожденная гипергеомет-

* Член рассеяния, фигурирующий в формуле (1), здесь включен в перенормировку спектра поглощения акщептора [ ${ }^{2}$ ].

** Спектр поглощения акцептора $\varkappa_{A}(\omega)$ аппроксимирован гауссианом. 


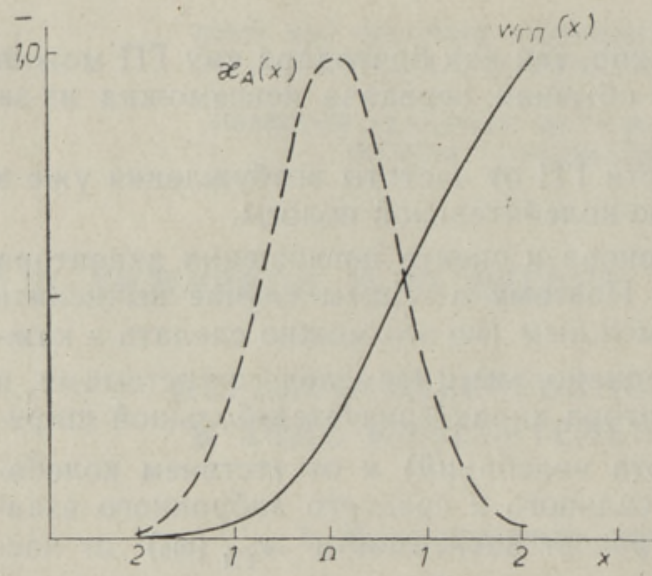

Рис. 1. Зависимость вероятности горячей передачи электронного возбуждения $w_{\Gamma п}$ от частоты возбуждения $x$ в случае медленной релаксации, рассчитанная по формуле (4) $\left(u^{2}=\right.$ $=0,5) ; x_{A}(x)-$ спектр поглощения акцептора.

рическая функция. Формула (4) дает быстрый монотонный рост $w_{г п}$ с увеличением $\omega_{0}$ во всем спектре

(рис. 1).

2. Быстрая колебательная релаксация в доноре. В этом случае зависимость $w_{г п}$ от частоты возбуждения несколько иная: огибающая спектра ГЛ в коротковолновой области падает по закону $\left[{ }^{3}\right]$

$$
I_{\text {гл }}\left(\omega_{0}, \omega\right) \sim\left(\omega_{0}^{\prime}-\omega\right)^{-1 / 2},
$$

где $\omega_{0}^{\prime}=\omega_{0}-\left(\omega_{0}-\omega_{D}\right)^{2} / 4 S_{D} \bar{\omega} \simeq \omega_{0} \quad\left(\omega_{D}-\right.$ частота максимума полосы поглощения донора). В результате зависимость вероятности соответствующей ГП от частоты возбуждения оказывается равной

$$
\begin{gathered}
w_{\Gamma \Pi}\left(\omega_{0}\right) \sim u^{2} \mathrm{e}^{-x^{2}} \int_{0}^{\infty} d y \frac{1}{\sqrt{y}} e^{2 x y-y^{2}}= \\
=u^{2} \frac{\sqrt{\pi}}{\sqrt{2}} \mathrm{e}^{-x^{2}}\left[\frac{\Gamma(1 / 2)}{\Gamma(3 / 4)} \Phi\left(\frac{1}{4} ; \frac{1}{2} ; x^{2}\right)-x \frac{\Gamma(-1 / 2)}{\Gamma(1 / 4)} \Phi\left(\frac{3}{4} ; \frac{3}{2} ; x^{2}\right)\right] .
\end{gathered}
$$

Эта зависимость изображена на рис. 2. Сравнение рис. 1 и 2 показывает, что зависимости $w_{г п}\left(\omega_{0}\right)$ от $\omega_{0}$ в обоих случаях похожи. Основное различие - поведение на коротковолновом краю полосы поглощения акцептора. ГП с такого типа зависимостями от $\omega_{0}$ наблюдалась: 1) в

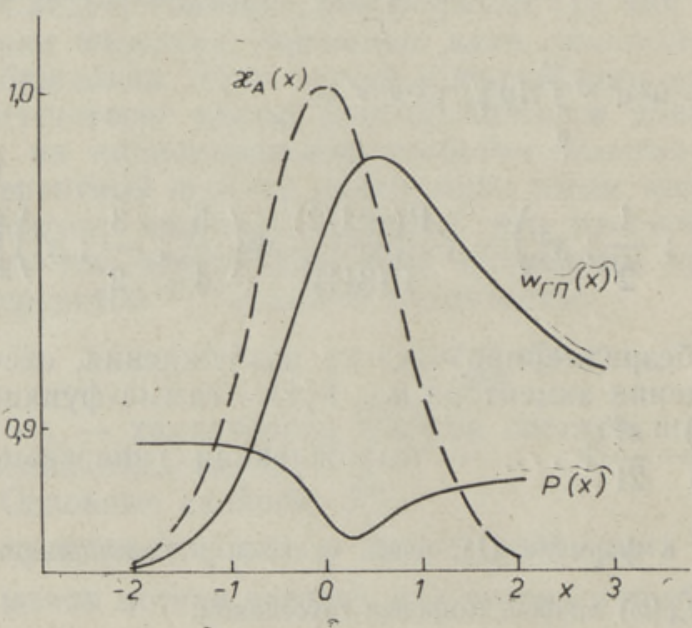

горячем спин-флипе в $F$-центрах $\left[{ }^{6}\right]$; 2) в горячей деполяризации люминесценции $\left[{ }^{7}\right]$; 3) в переносе электронного возбуждения с высших возбужденных уровней в органических соединениях (см. напр. $\left[{ }^{8,9}\right]$ ).

Рис. 2. $w_{\text {гп }}(x)$ в случае быстрой релаксации, рассчитанная по формуле (6) $\left(u^{2}=0,5\right) ; P(x)-$ поляризация люминесценции как функция частоты возбуждения (см. [3]). 
Рис. 3. Деполяризация многофононного резонансного комбинационного рассеяния как функция от частоты рассеяния $z$, рассчитанная по формуле (8) для частот возбуждения $z_{0}=$ $=1 \quad(1), 1 / 2 \quad(2), 0 \quad(3),-1 / 2$ (4) и -1 (5).

Обратим внимание на то, что ГП объясняет также зависимость степени поляризации РВС от частоты воз-

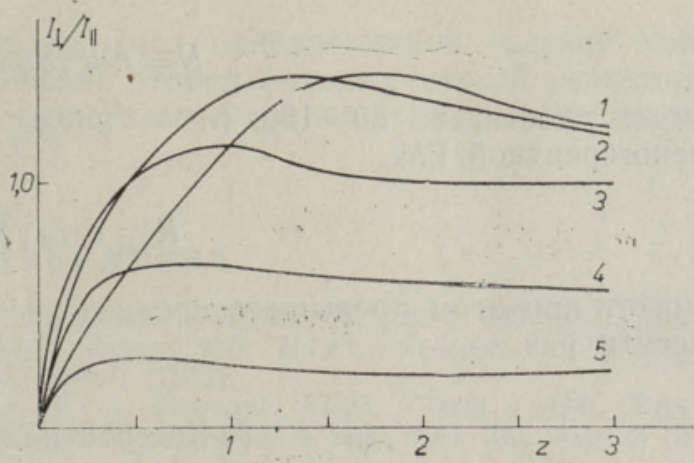
буждения (случай внутри-

центровой передачи энергии). В частности, внутрицентровая ГП между разными компонентами вырожденного возбужденного электронного состояния приводит к зависимости степени поляризации ОЛ от частоты возбуждения $\left[{ }^{3}\right]$ (см. рис. 2). Кроме того, зависимссть степени поляризации многофононного резонансного комбинационного рассеяния (PKP) от частоты рассеяния $\Omega$ может быть также объяснена ГП. Действительно, огибающая многофононного РКР на частоте $\Omega$ определяется интенсивностью ГЛ в этой частоте. Если степень деполяризации не велика, то в случае быстрой релаксации эта интенсивность в параллельной поляризации

$$
I_{\rrbracket} \sim\left(\omega_{0}-\Omega\right)^{-1 / 2}
$$

а в перпендикулярной

$$
I_{\perp} \sim \int_{\Omega}^{\omega_{0}} x(\omega) d \omega /\left[\left(\omega_{0}-\omega\right)(\omega-\Omega)\right]^{1 / 2}
$$

Здесь интеграл по $\omega$ суммирует процессы, в которых ГП произошла на разных этапах движения конфигурационной координаты (учитывается лишь первый полупериод колебаний). В результате зависимость отношения $I_{\perp} / I_{\|}$от частот возбуждения и рассеяния оказывается равной (см. рис. 3)

$$
I_{\perp} I_{\|} \sim \sqrt{z} \mathrm{e}^{-z_{0}^{2}} \int_{0}^{1} d x \frac{\mathrm{e}^{-z^{2} x^{2}+2 z z_{0} x}}{\sqrt{x(1-x)}}
$$

где $z=\left(\omega_{0}-\Omega\right) / \sigma \sqrt{2}, z_{0}=\left(\omega_{0}-\omega_{\mathrm{M}}\right) / \sigma \sqrt{2}, \omega_{\mathrm{M}}-$ частота максимума полосы поглощения.

\section{2. Некогерентная горячая миграция}

Если взаимодействие центров достаточно велико, но $u<\sigma_{A}$, может произойти многократная некогерентная ГП еще до окончания колебательной релаксации, т. е. может произойти некогерентная горячая миграция (ГМ) $\left[{ }^{10}\right]$. Интересно отметить, что даже в случае быстрой релаксации такой процесс актуален, причем сама ГМ несколько замедляет релаксацию, усиливая ГЛ. Время некогерентной ГМ

$$
t_{\Gamma \mathrm{M}} \sim \sqrt{\bar{N}} t_{\Gamma \Pi} \sim \sigma / \hbar u^{2}
$$

где число прыжков 


$$
N=n^{2} u^{4} / S \sigma \omega^{3}
$$

может достигать 50-100 ( $n-$ число ближайших соседей). Радиус некогерентной ГМ

$$
R_{\Gamma M} \sim r_{0} \sqrt{N}
$$

может при этом превышать среднее расстояние между центрами $r_{0}$ до десяти раз.

\section{3. Когерентная ГП}

При сильном взаимодействии молекул

$$
u>\Gamma
$$

(Г - ширина резонансного вибронного уровня) имеет место когерентная ГП. В этом случае, как уже отмечалось, система сильно перестраивается: состояния донора и акцептора коллективизируются. Возникающие коллективные вибронные состояния релаксируют с испусканием фононов. Учитывая диффузионный характер движения возбуждения по системе, радиус ГП в этом случае можно оценить по формуле

$$
R_{\text {гп }} \sim\left(L v_{0} t_{\text {столкн }}\right)^{1 / 2} \sim r_{0} \frac{u^{2}}{\sigma^{2}}\left(\frac{u}{\bar{\omega}}\right)^{1 / 2} \sim \frac{r_{0}}{S(2 \bar{n}+1)}\left(\frac{u}{\bar{\omega}}\right)^{5 / 2}
$$

где $v_{0} \sim r_{0} u / \hbar-$ средняя скорость, $\bar{n}=\left[\exp \left(\bar{\hbar}_{\omega} / k T\right)-1\right]^{-1}, \quad L=$ $=v_{0} t_{\text {столкн }}$ - длина свободного пробега, $t_{\text {столкн }}-$ характерное время рассеяния коллективного возбуждения на фононе. По порядку величины

$$
t_{\text {столкн }} \sim \hbar u / \sigma^{2} \sim t_{\mathrm{rп}} \bar{\omega} / u,
$$

где

$$
t_{\text {гп }} \sim \hbar u^{2} / \bar{\omega} \sigma^{2}
$$

- время когерентной ГП.

Из формулы (12) следует, что при достаточно высокой концентрации центров радиус когерентной ГП может достигать сотен и тысяч ангстрем. Отметим, что указанные выше три основных свойства ГП высокая скорость, малая потеря энергии, сильная зависимость от частоты возбуждения - относятся и к когерентной ГП.

\section{4. Заключение}

Может ли механизм ГП иметь место при переносе энергии между молекулами хлорофилла; например, в фотосинтетической единице? Этот вопрос еще ждет своего ответа. Во всяком случае отмеченные выше общие свойства ГП - высокая скорость процесса и отсутствие заметной потери энергии - не противоречат известным данным по переносу возбуждения между молекулами хлорофилла in vivo. Весьма важно также, что эффективность ГП (в особенности когерентной ГП) достаточна для обеспечения переноса энергии на требуемые расстояния $\sim 100-200 \AA$ (см. формулы (11) и (12)). Отметим, что в пользу механизма ГП свидетельствует также наблюдавшаяся в [11] сильная зависимость скорости фотосинтеза от частоты возбуждения в области длинноволновой 
полосы поглощения хлорофилла $a$ (при одновременной зеленой подсветке, необходимой для обеспечения второй фотохимической реакции). Эта зависимость качественно согласуется с приведенными выше теоретическими зависимостями для ГП.

\section{ЛИТЕРАТУРА}

1. Тех вер И. Ю., Хи жн яков В. В., Письма в ЖЭТФ, 19, вып. $6,338-342$ (1974).

2. Хи жн яков В. В., Препринт FAI-11, Тарту, 1972; Hizhnyakov, V. V., Phys. status solidi (b), 51, № 2, K117-K119 (1972)

3. Hizhnyakov, V. V., Tehver, I. J., Preprint FI-31, Tartu, 1974; Tex в е р И. Ю., Хи жняков В. В., Ж. эксперим. и теор. физ., 69, вып. 2 (8), $599-610$ (1975).

4. Förster, Th., Ann. Phys., ser. 6, 2, 55-75 (1948).

5. Dexter, D. L.. Phys. status solidi (b), 51, № 2, 571-578 (1972).

6. Лий дья Г., Соови к Т., Физ. твердого тела, 21, вып. 8, 2377-2383 (1979).

7. Fukuda, A., Makishima, S., Maruchi, T., Onaka, R., J. Phys. Chem. Solids, 28, № 9, 1763-1780 (1967).

8. Бендерский В. А., Брикенштейн В. Х., Филиппов П. Г, Яцен к о А. В., Оптика и спектроскопия, 45, вып. 3, 512-517 (1978).

9. Богд а н о в В. Д., К лочков В. П., Оптика и спектроскопия, 48, вып. 4, 34-48 (1980).

10. Hizhnyakov, V. V., Phvs. status solidi (b), 76, № 1, K69-K72 (1976).

11. Х и т О., Фотосинтез, М., «Мир», 1972.

\section{Институт физики \\ Академии наук Эстонской ССР}

\section{V. HIZNJAKOV, I. J. TEHVER}

\section{ELEKTRONERGASTUSE OLEKANNE VONKERELAKSATSIOONI KAIGUS}

Artikkel käsitleb elektronergastuse koherentset ja mittekoherentset ülekannet relakseerumata võnkeolekus. On esitatud ülekande raadiuse ja aja valemid mõlemal juhul, samuti elektronergastuse energia ülekande tõenäosuse sōltuvus ergastussagedusest. On vaadeldud energiaülekande mehhanismi osa luminestsentsi ja resonantse kombinatsioonhajumise depolarisatsioonis ning hinnatud tema võimalikku osatähtsust energia ülekandes klorofülli molekulide vahel.

\section{V. HIZHNYAKOV, I. J. TEHVER}

\section{TRANSFER OF ELECTRONIC EXCITATION DURING VIBRATIONAL RELAXATION}

The coherent and incoherent transfer of electronic excitation in nonrelaxed vibrational state is considered. Formulae for the transfer radius and time in both cases are obtained, the dependence of the transfer probability on the exciting frequency is calculated. The role of this energy transfer mechanism in the depolarization of luminescence and of resonant Raman scattering is discussed. The possibility of such a transfer between chlorophyll molecules is supposed. 